\title{
LVIII. Sertum antillanum. VII.
}

Auctore Ign. Lisban.

155. Carludovlos,rigida (Aubl.) Lirb., comb. nov. - Pothas rigida Aubl. Guian. II (1776) p. 839. - Salmia palmaetolia Willd. in Mag. Gos. Naturf. Proundo Berlin V (1811) p. 101. - Carludovica Phmerii Kunth Enum. III 11841) p. 106; Duss Flor. Ant. franf. p. 484 . - C. palmifolia 0. Ktze. Rov. Il (1891) p. 738; Baill. Hist. Plant. XIII (1895) p. 421. - Arum hederaceum, faliis bissectis, rigidis et sulcatis Plum. Descr. Plant. Amxx. (1693) p. 43 tab. 51 igg. $f$ ot 59. - Ailo a mouchos v. Languo a booul v. Petit balisior Guad., Zoll Mouche Domin., Cachibou Martin.

Radiculis adventivis in arboribus altissimis usque ad apicem scandens.

Hab. in Guadoloupo in sylvis humidis $300-1000 \mathrm{~m}$ frequentissima, 0. g. in sylvis Bains-Jaunes, m. Jan.-Jun. nor.: Duss no. 3312, 3807, 4199. - Dominica: Kamage. - Martiniquo in sylvis ad Cabestorro ad'parochiam Sainte Marie versus: ex Plumier, in omnibus sylvis magnis 300-900 m frequens: Duss no. 2008, Hahn no. 1019. - St. Vincent in monte St. Andrews: Eggors no. 6817. - Gronada: Sherring, in silvis ad montem Felix $530 \mathrm{~m}$ : Eggors no. 6089. - Trinidad?: Bot. Gard. Ilorb. no. 2233.

156. Dioscores altissima Lam. Enc. III (1789) p. 231 ; Kunth Enum. V p. 418; : Eggers Flor. St. Croix and Virg. Isl. p. 110. - D. chondracarpa Griseb.! in Mart. Flor. Bras. III. I. (1842) p. 34. - D. alata var. altissima Griseb. Flor. (1864) p. 587 (saltem quosd syn. Plum.). - Pdygomatwm scandens, altissimum. foliis tamni Plum. Cat. pl. Amer. (1708) p. 1 ot od. Burm. p. 108 tab. 117 fig. 2. - Ignamo bătard, Igname grand bois. Ronice Guad.

Volubilis, in arbores altissimas scandens. Radix tuboriformis horizontalis lignosa, intus nava. Propagatio fit bulbillis aẽrois docidentibus ot radicantibus. Inflorescentia dependentes. Flor. April., Majo, Aug., Sept. (omnia ex Duss).

Hab.? in insulis Is anicis: ex Eggers. - Guadoloupo: Duchassaing. ad Baie Mahault (Bois do la Digue): Duss no. 3545, in Camp Jacob ad Rividre.Noire: Duss no. 3872. - Martinique: Plumier (ex Lam.). Tobago. in convalli numinis superioris Great Dog River: Eggers no. 5772. - Praeteres: Brasilia (civit. Rio de Janoiro et Paraná).

Anm. Bereits Lline und ihm folgend der Monograph der Familie R. Knuth hatten die genannten Pflanzen von den kleinen Intillen im Herbar Krug et Urban als $D$. chondrocarpa Griseb. bestimmt, also mit einer bisher nur aus dem südlicheren Brasilien bekannten Art identifziert. Auch ich habe mich nach Besichtigung des Originalexemplars von Grisobach überzeugt, dass die Cbereinstimmung in Stacholbildung, Hlattorm, Blütenatänden eine vollkommene ist; die Früchte sind allerdings von Westindien nicht bekannt. 
Diese Art hat also eine zhnliche pflanzengeographisch so rätselhafte Verbreitung wio die Loranthacee Eubrachion ambigum (Hook. et Arn.) Engl. (Jamaica, sưdl. Brasilien, Uruguay und Argentina) und Phyllostylon brasiliense Capanema (Cuba, Hispaniola, südl. Brasilien und Paraguay). Dass sie aus Brasilien zu .Kulturzwecken nach den Antillen übergeführt sei, ist ganz ausgeschlossen, einmal weil sie nur wild beobachtet wurdo und von den Knollen gar keine Verwendung bekannt ist, sodann weil eine derartige Überführung von Pflanzen aus Brasilien nach den Antillen niemals stattgefunden hat (im. Gegensatz zu der Bereicherung westindischer Gärten durch altweltliche Kulturpflanzen aus Ostindien und den ostafrikanischen Inseln) und endlich, weil sie, wie wir sahen, bereits um 1700 von Plumier auf Martinique beobachtet wurde.

Der älteste Name für diese Pflanze ist aber $D$. altissima Lam., eine den Botanikern bisher ganz unbekannt gebliebene Art, gegründet auf Plumiers Beschreibung (Manuscript vol. IIl t. 144 im Muséum d'hist. nat. zu Paris) und auf seine von Burman publizierte Abbildung. Der Wuchs (altissime scandens bei Plumier und Duss), die (nicht immer genau) gegenständigen Blätter, die Blattform, die langen hängenden Ähren und besonders die Stipularstacheln, ausserdem auch das Vaterland (allerdings auf Martinique von neueren Botanikern nicht gesammelt, aber wohl auf Guadeloupe) lassen keinen Zweifel an der spezifischen Übereinstimmung.

Die jungen aus den Knollen hervorgehenden Pflanzen haben durchweg abwechselnde Blätter. Die älteren Zweige sind mit sehr zahlreichen dreieckigen, von der Seite her stark zusammengedrückten flachen Stacheln besetzt.

157. Piper constanzanum (C. DC.) Urb., spec. nov. - P. tenuiamentum O. DC. var. constanzanum C. DC. in Urb. Symb. ant. VII (1912) p. 184.

Frutex amplus valde fragilis (ex coll.). Ramuli juniores pube brevi crispa obsessi. Stipulae in unam oppositifoliam gemmam amplectentem coalitae, ovatae, superne plicatae el acuminatae, usque $12 \mathrm{~mm}$ longae, membranaceae, dorso medio pilosae. Folia petiolis $8-2 \mathrm{~mm}$ longis ad basin paullo dilatatis praedita, ovato-elliptica v. rhombeo-elliptica, inferne subcuneata, basi inaequilatera, latere altero quam alterum subrectum $2-3 \mathrm{~mm}$ longius descendente, apice acuminata, $9-15 \mathrm{~cm}$ longa, $3-7 \mathrm{~cm}$ lata, nervis lateralibus 4-5 pinnatis sub angulo $20-30^{\circ}$ e medio in parte ejus inferiore abeuntibus, supra parce et brevissime pilosa, subtus ad nervos brevissime strigoso-pilosa, initio membranacea, posterius areolatorugosa. Pedunculus $7-9 \mathrm{~mm}$ longus; spica cr. $8 \mathrm{~cm}$ longa, $2 \mathrm{~mm}$ crassa; bructeae pelta triangularis brevissime pilosa. Stamina ad basin ovarii inserta; antherae quadrato-rotundatae. Ovarium ovato-globosum glabrum; stigmata 3 brevia linearia recurva.

Hab. in Sto. Domingo prope Constanza in sylva vallis Tireo $1170 \mathrm{~m}$, m. Majo flor.: v. Türckheim no. 3237. 
Obs. P. tenwiamentum C. DC. (e Trinidad) differt foliis anguste ovatis v. ovato-ellipticis, basi tota (utroque baseos 'atere) rotundatis, postremo quoque membranaceis non areolatis, spicis $10-12 \mathrm{~mm}$ longe pedunculatis. - P. jamaicense (Griseb.) C. DC. quoque ad nostram speciem valde accedit.

158. Ampolocera oronulata Urb., spec. nov.

Verisimiliter fruticosa. Rami vetustiores teretes, lenticellis minutis orbicularibus obsessi; ramuli hornotini basi squamas incrassatas snbrotundas v. obtuse triangulares gerentes, breviter et tenuiter pilosuli et hoc tempore jam lenticellosi. Stipulae liberae, inferiores oblongae, superiores lenceolato-lineares, $1,5-2,6 \mathrm{~mm}$ longae, deciduae. Folis alterna, 2-3 mm longe petiolata, ovata v. ovato-elliptica, basi obtusa v. rotundata, apice acute acuminata, $4-7 \mathrm{~cm}$ longa, $2-3 \mathrm{~cm}$ lata, pennatinervia, nervis supra prominentibus et utrinque bene reticulato-anastomosantibus, margine toto dense crenulata, crenulis apice incurvis, supra glabra nitida, subtus praesertim secus nervam medium brevissime et tenuissime puberula, tenuiter chartacea, in sicco nigrescentia. Flores (valde juveniles tantum visi) plures in cyathulum ad nodos ramorum vestustos subsessilem v. usque $2 \mathrm{~mm}$ longe stipitatum $\mathrm{cr} .2,5 \mathrm{~mm}$ crassum, 1,5 mm longum corticoso-induratum collecti, bracteis 2 ovatis acutis intus ad basin breviter pilosis inclusi. Alabastra ovalia pedicellata. Perianthii phylla 5 in aestivatione imbricata, glabra. Stamina 10 basi perianthii affixa; antherae subquadrato-orbiculares, longitrorsum birimosae. Ovarium superum glabrum; stigmata 2.

Hab. in Cuba: Ramon de la Sagra no. 413.

Obs. I. In cyathulo fila nonnulla rigida inter alabastra prodeuntia usque $5 \mathrm{~mm}$ longa inveniuntur; an pedicelli florum vetustorum delapsorum?

Obs. II. A. cubensis Griseb. ramis hornotinis minutissime patentipilosis, foliis 3-6. mm longe petiolatis, basi obsolete excisis, integris v. in specim. Doming. superne crenis parcissimis obsitis, squamis involucrantibus liberis nec in cyathulum connatis, perianthii segmentis 4 et verisimiliter aliis characteribus floris fructusque differt; $A$. Ruizii Kl. (e Pera) foliis basi cunesto-angustatis parce dentatis $v$. subintegris, ovario dense tomentoso recedit.

Obs. III. Si nostra species re vera ad genus Ampelocerae pertineat, materies melior in futuro demonstrabit.

159. Ranunculus recurvatus Poir.; Fawc. et Rendle Flor. Jam. III p. 188.

Fawcett und Rendle führen für Jamaica $R$. recurvatus Poir. mit leaves 3-cloft und eine Varietät tropicus (Griseb.) Fawc. et Rendle ( $R$. repens var. tropicus Griseb. Flor. p. 1, R. cubensis Griseb. Cat. p. 1) mit leaves 3-foliate or 3-sect auf. Es ist mir ganz unverständlich, wie sie dazu kommen, die beiden Gris ebach schen Arten, die ganz verschiedenen Gruppen angehören, miteinander zu identifizieren. Die erstere wurde von Macfadyen Flor. Jam. p. 3 als $R$. repens bestimmt mit der Bemerkung: This is evidently an introduced plant which has become 
naturalized from the garden of the late Mr. Matthew Wallen at Coldspring, and is now very plentiful in the above locality. Diese Pflanze von Macfadyen hat Grisebach gesehen und zu seiner Varietat tropicus von $\boldsymbol{R}$. repens L, gezogen, indem or sie für ganz identisch mit $\boldsymbol{R}$. praemorsus H. B. K. aus den Gebirgen des tropischen Südemerikas erklärte. Sowohl aus der Bestimmung von Macfadyen wio aus den Noten von Grisebach Flor. p. 1 geht hervor, dass wir darin einen echten $R$. repens L. (wie Eggers no. 353b und Harris no. 6303) oder weniger wahrscheinlich eine ihm sehr ähnliche, aber durch den zurückgeschlagenen Kelch abweichende, auf der Insel seitdem nicht wieder gefundene Pflanze zu erkenncn haben, die ein länger gestieltes Nittelblättchen und 5-10 verhältnismässig grosse; Blumenblätter besitzt. Mag nun Grisebach der Pflanze Macfadyens mit Recht oder mit Unrecht einen Calyx reflexed zuschreiben, jedenfalls ist $R$. repens var. tropicus Griseb. nach der Beschroibung und den Synonymen $2 u$. praemorsus H. B. K. zu ziehen. - $\boldsymbol{R}$. cubensis Griseb., der in der Tat dem nordamerikanischen $R$. recurvatus Poir. sehr nahesteht und mit diesem in Westindien auch durch Uebergangsformen verbunden scheint, hat dagegen ungestielte Mittelblättchen und 4-5 kleine den Kelch nur wenig uberragende Blumenblätter. Offenbar hat Macfadyen nach der Veröffentlichung des ersten Bandes seiner Flora of Jamaica (1837) auf der Insel auch den echten $R$. cubensis entdeckt, da ein solches Exemplar von den obengenannten Autoren zitiert wird; ich sah aus Jamaica nur den typischen $R$. recurvatus Poir. (Harris no. 5891 und 6304, lotztere Nummer gemischt mit dem eingeschleppten $R$. parviflorus L.), nicht den $R$. cubensis Griseb.

160. Canavalia maritima (Aubl.) Thou. in Desv. Journ. Bot. I (1813) p. 80. - Dolichos maritimus Aubl. Guian. II (1775) p. 765. - D. lineatus Thunbg. Flor. Jap. (1784) p. 280. - $D$. obtusifolius var, $\beta$ Lam. Enc. II (1786) p. 295. - Canavalia obtusifolia P. DC. Prodr. II (1825) p. 404 (excl. syn. Lam. genuino); Bonth. in Mart. Flor. Bras. XV. I p. 178 tab. 48; Griseb. Flor. p. 197. - C. lineata P. DC. 1. c. - Phaseolus maritinus, fructu duro, semine variegato Plum. Cat. pl. amer. (1703) p. 8 et (ex Aubl. et Lam.) Msc. vol. Il tab. 99.

Hab. in regionibus tropicis utriusque orbis, in litoralibus repens.

Anm. Bis in die neuere Zeit wurde diese an den Meeresküsten der Tropen verbreitete Art mit dem irrtümlichen Namen Canavalia obtusifolia (Lam.) P. DC. bezeichnet. Die Lamarcksche Art, die sich auf Plumiers Phaseolus amplissimus, siliqua maxinua, fructu coccineo, duro (Msc. vol. II tab. 86) gründet, hat $6-8$ Zoll lange, 11/2 Zoll breite Hülsen, mehr oder weniger lebhaft rote Samen und klettert über die höchsten Bäume; ob sie mit meiner $C$. rusiosperma zusammenfällt, was nicht unwahrscheinlich ist, muss der Vergleich der Exemplare mit Plumiers leider nicht veröffentlichter Abbildung ergeben. Dagegen gehört der Dolichos obtusifolius var. $\beta$ Lam. nach der kurzen Beschreibung Plumiers und Lamarcks mit Sicherheit hierher.

Aus Prioritätsgründen hat man in neuester Zeit den Namen Canavalia 
lineata (Thunbg. 1784) P. DC. vorangestellt. Allein es existiert in Dolichos maritimus Aubl, (1775) ein noch älterer Name, der auf dieselbe Plumiersche Art und Abbildung begründet ist. Wenn nun auch Petit-Thouars, der übrigens schon auf die Unterschiede gegenüber der Lamarckschen Art hinwies, aber von dem Vorhandensein jenes äteren Binoms keine Kenntnis hatte, den Namen Canavali maritima Thou. auf Grund von ihm studierter Pflanzen von Madagascar und Indien selbständig bildete, so muss man doch wohl, da die westindische ùnd altweltliche Pflanze dieselbe Art darstellen, ihrem Namen die Autorität (Aubl.) Petit-Thouars hinzufügen.

161. Casfola Turpin (Neocastela Small und Castelaria Small).

Small taufte in der North Amer. Flor. Vol. 25 part 3 (1911) p. 230 die alte Gattung Castela Turp. (1806), weil bereits eine Castelia Cav. (1801, übrigens ein Synonym von Priva) existiere, in Neocastela (mit $N$. depressa) um und spaltete von ihr als neues Genus Castelaria (mit allen übrigen Arten) ab. Diese Zersplitterung bietet ein schönes Beispiel für die Leichtfertigkeit, mit der in der New Yorker schule bei der Aufstellung neuer Gattungen verfahren wird. Die Unterschiede sind nach Small l. c. p. 227:

Neocastela: Styles united; anthers apiculate at the apex.

Castelaria: Styles distinct; anthers notched at the apex.

Bei der ersteren sind nach der Turpinschen Abbildung in der Tat die Griffel bis zur Spitze vereinigt; die Art selbst ist noch nicht wiedergefunden und das Original scheint auch nicht wieder untersucht worden zu sein. Bei Castelaria gibt es aber, was von $\mathrm{Small}$ einfach tot geschwiegen wird, auch Arten, bei denen die Griffel nicht distinct, sondern unterwärts verwachsen sind, wie bereits Planchon, Bentham et Hooker und Engler hervorhoben, und wie ich es auch bei meiner C. macrophylla (Symb. V p. 377) beschrieb. Was aber die von den Antheren hergenommenen Unterschiede betrifft, so stehen diese in direktem Gegensatz zu den in den Gattungsdiagnosen S. 230 aufgefuhrten Charakteren. Hier werden auch (unter Castelaria) die an thers apiculate at the apex verwandelt in anthers not notched, sometimes apiculate at the apex. Mag nun auch bei dieser Vertauschung von Seiten Smalls ein Lapsus calami volliegen, so ergibt sich aus dem vorstehenden jedenfalls die Geringwertigkeit auch der Merkmale in den Antheren. - Die zweite von Turpin aufgestellte Art: C. erecla wurde nach einem Exemplare des Herb. L. C. Richard beschrieben, das dieser selbst auf der Insel Antigua gesammelt hatte; Small nennt aber, der irrtümlichen Angabe Pla nch on s folgend, als Type locality Santo Domingo und erwähnt die Insel Antigua überhaupt nicht.

Ausser durch die Blattform unterscheidet Turpin seine beiden Castela-Arten durch die Stellung der Dornen: C. depressa spinis axillaribus, c. erecta spinis infra-axillaribus. Wenn dio Sache sich so verhielte, würden das sehr beachtenswerte Charaktere sein. In Wirklichkeit ist aber die Differenz in der Stellung der Dornen nicht so gross. Diese

Bopertorium specierum novarum, XV. (31. III. 1918.) 
sind umgewandelte Seitenzwoige aus der Achsel von Laubblättern, bald surz und dann oft die Spuren ron Blattschüppchen tragend, bald mehr oder weniger verlängert und mit Laubblättern besetzt; in letzterem Falle erscheinen sie als Endigungen von Seitenzweigen. So bei Castela depressa Turp., C. Nicholsonii Hook., C. Tweedii Planch., C. macrophylla Urb. Bei C. erecta Turp. dagegen findet man als Tragblatt des Dorns nur selten ein winziges Blättchen; gewöhnlich ist dies völlig unterdrückt und durch einen kleinen Wulst oder eine Schwiele vertreten; dafür hat aber die in der Achsel des Dorns stehende Knospe (oberstăndige Beiknospe) gewöhnlich ein, seltener zwei normale Blätter entwickelt, die physiologisch die Funktion der unterdrückten primären Blätter ausüben. So kommt es, dass hier bei oberflächlicher Betrachtung die Dornen hypophyll zu stehen scheinén. Systematisch interessant ist es nun zu sehen, dass die monotypische Neocastela Small sich in dieser Beziehung wie die meisten Castelaria-Arten verhält, während umgekehrt von letzteren die C. erecta die Mutterblätter der Dornen unterdrückt. Es gibt aber auch eine Art, $C$. retusa Liebm., bei der an demselben Zweige das Tragblatt der Dornen bald voll entwickelt, bald abortiert ist. Dies interessante morphologische Verhalten von den Dornen zu den Blättern ist bisher von niemandem, auch nicht von Small, erörtert worden.

162. Malpighia domingonsis Small in North Amer. Flor. 25. II (1910) p. 156 (excl. syn. L. et Juss.). - M. urens Griseb. in Linnaea XXII (1849) p. 1 (non L.); Niedenzu Gen. Malp. (1899) p. 9 (var. typica Ndz.).

Der Name $M$. urens ist von den Autoren für sehr verschiedene Pflanzen gebraucht worden. Es fragt sich nun, was Linné in der ersten Ausgabe der Species plant. darunter verstanden hat. Da seine Diagnose keine Anhaltspunkte zur Beurteilung dieser Frage bietet, so haben wir die von ihm zitierten Synonyma einer kritischen Ṕrüfung zu unterwerfen. Das erste ist Malpighia angustifolia èt latifolia, fructu (sphalmate pro folio) subtus spinoso Plum. Gen. (1703) p. 46. Hier hat Linné zwei Plumiersche Arten: $M$. angusti-folia, folio swbtus spinoso und $M$. lati-folia, folio subtus spinoso irrtümlicherweise miteinender vereinigt. In der zweiten Ausgabe (1762) wird die erstere, die schon kurz vorher (1760) von Jacquin als $M$. linearis beschrieben war, als besondere Art unter dem Namen $M$. angustifolia wieder ausgeschieden; sie ist eine Bewohnerin der Kleinen Antillen, kommt also für Hispaniola nicht in Betracht. Die andere Art lässt sich aus der ersten Ausgabe der Species nicht deuten, wohl aber aus der zwoiten, in welcher zu der M. Latifolia, folio subtus spinoso die mittlerweile (1758) von Burman publizierte Plumiersche Tafel 167 fig. 1 zitiert wird. Diese Figur, die Plumier, wie I ussiou mitteilt (Arch. Mus. Par. III p. 257), nach einer Pflanze von St. Croix angefertigt hat, stimmt vollkommen überein mit $M$. fucata Ker var. elliptica $N d z$. von Portorico und den Kleinen Antillen (z. B. St. Croix: A. E. Ricksecker no. 339). Damit scheint die Frage nach der Bedeutung der beiden Plumierschen Arten gelöst zu sein. Nun stellte aber Jussieu aus den Manuskripten Plumiers fest, dass dieser die $M$. angusti-folia zuerst 
M. Latifolia folio subtus spinoso genannt habe und dass $z$ a dieser die Tafel $167 \mathrm{flg} .1$ gehöre, und identiflzierte sie mit M. urens L., bzw. mit der var. infestissima (Rich.) (nach meiner Meinung, wie gesagt, ist es aber $\boldsymbol{M}$. fucata Ker), während die zweite Art ursprünglich $\boldsymbol{M}$. alia latifolia folio subtus piloso ad nodos florida hiess und von ihm für M. fucata Ker erklärt wurde. Wie dem auch sein möge, jedenfalls kommt bei den beiden PJumierschen Arfen keine Hispaniola-Pflanze in Betracht.

Das zweite Zitat bei Linné: Mespilus Americana, folio lata subtus spinoso, fructu rubro Tourn. Inst. p. 642 bezieht sich auf eine der beiden vorbingenannten Arten Plumiers.

Das dritte Zitat: Arbor baccifera, folio oblongo etc. Sloane Jam. p. 172 et Hist. II p. 106 t. 207 f. 3 betrifft eine Jamaica-Pflanze, die von Niedenzu l. c. p. 13 zu M. martinicensis Jacq. gezogen, von Small b. c. p. 158 als $M$. urens $L$. aufgeführt wurde.

Aus den von Linné aufgeführten Synonymen ergibt sich, dass die der Insel Hispaniola eigentümliche, von Niedenzu als $M$. urens bezeichnete Art nicht darin enthalten ist und daher einen neuen Namen orhalten musste, vorausgesetzt, dass das Exemplar des Herbars Linné nicht etwa hierher gehört und dass dieses seiner Beschreibung wirklich zugrunde gelegen hat. Jussieu hat zwar das Original gesehen und es zu M. urens zitiert; da aber auch er noch unter diesem Namen verschiedene Arten zusammenfasst, so bedarf es einer neuen sorgtältigen Untersuchung des Typus.

Nach Niedenzu in Arb. Bot. Inst. Braunsberg V (1914) p. $3 b$ soll die Art auch auf Cuba von $R$. de la Sagra gefunden sein.

163. Malpighia mogacantha (Juss.) Urb., spec. nov. - M. urens L. var. megacantha A. Juss.! Mon. Malp. in Arch. Mus. Paris III (1843) p. 260 (s. i. II p. 6); Walp. Rep. V p. 151; Niedenzu Gen. Malp. (1899) p. 10. - M. domingensis Small, in North Amer. Flor. 25. II (1910) p. 156 (p. p., cum?).

Frutex $0,5 \mathrm{~m}$ altus. Fl. m. Nov.

Patria non adjecta: Herb. Vaillant (mus. Paris.). - Haiti propo Pétionville in montibus $800 \mathrm{~m}$ : Picarda no. 1496.

Anm. Die Uebereinstimmung der beiden zitierten Exemplare ist eine vollkommene. Die doppelt kleineren viel dünneren lang zugespitzten Blätter, die auffällig langen (bis $11 \mathrm{~mm}$ langen) zahlreichen Malpighiaceөnhaare auf deren Unterseite, die kleineren Blüten und besonders die geringere Anzahl der Kelchdrüsen (6-8 statt 10) entfernen sie soweit von M. domingensis Small dass ihnen das Artrecht zuerkannt werden muss.

164. Malpighia aquifolia, amplioribus folis Plum. Cat. Amer. (1703) p. 22 in add. et ed. Burm. fasc. VII (1758) p. 160 tab. 167 fig. 2. Falentinia ilicifolia Sw. Prodr. (1788) p. 63 et Flor. II p. 689 (quoad sjn. Plum.); Poir. in Lam. Enc. VIII p. 293. - Chicharron Doming.

Arbor sylvestris elata ligno durissimo (ex Türckh.). Flores ot fructus ignoti.

Hab. sine dubio in Haiti: Plumier. - Santo Domingo in prov. 
Barahona prope Barahona ad Maniel $600 \mathrm{~m}$ alt., m. Jan.: v. Türckheim no. 2787, prope Constanza $1190 \mathrm{~m}$ alt., m. Jun.: idem no. 3402 .

Anm. Schon Burman machte 1. c. darauf aufmerksam, dass diesor Baum (nicht Strauch, wie or ohne Grund schreibt), von dem Plumier bloss einen Blattzweig gezeichnet hatte, wegen der Alternanz der Blätter nicht zu der Gattung Malpighia gehören könne. So viel ich weiss, hat nur $\mathrm{S} w a r t z$ eine Deutung dieser Abbildung versucht, indem or sio zu seiner Valentinia ilicifalia zitierte. Allein sicher mit Unrecht; denn diese ist ein $0,6-5 \mathrm{~m}$ hoher Strauch mit kürzeren und breiteren vorn abgerundeten oder gestutzten Blättern. Nach meiner Meinung stellt die Plumiersche Figur die noch nicht beschriebene Art einer vielleicht neuen Gattung dar.

Die Türckhөimschen Exemplare stimmen nun riicksichtlich der Länge der Blattstiele, der Form und Grösse der Blätter, der Nervatur und Bezahnung so vollständig mit der zitierten Abbildung überein, dass ich überzeugt bin, dass wir darin die Pflanze Plumiers vor uns haben. An der Hand der genauen Standorte und des einheimischen Namens, der an beiden Lokalitäten der gleiche ist, wird es künftigen Sammlern gelingen, den Baum wioder aufzufinden und Blüten und Früchte zu erlangen, so dass die systematische Stellung ermittelt werden kann.

Ich will noch bemerken, dass die Pflanze mit der CombretaceonGattung Chicharronia A. Rich. von Cuba nichts zu tun hat, auch durch die Blätter von 'Trichilia cuneifolia (L.) Urb. leicht zu unterscheiden ist, die, wie Valentinia, auf Santo Domingo ebenfalls den Vernacular-Namen Chicharron führt.

165. Phyllanthus cornifolius H. B. K.: Nov. Gen. et Spec. II (1817) p. 115. - Asterandra cornifolia Kl. in Wiegm. Arch. VII (1841) p. 200. Phyllanthus grandifolius var. cornifolius Müll. Arg. in DC. Prodr. XV. II (1866) p. 329. - P. smilacifolius Griseb. Flor. (1864) p. 710 (nomen).

Folia ovato-elliptica v.' elliptico-oblonga, basi truncata v. subcordata. Pedicelli florum femin. 20-25 mm longi. Stigmata peltata triloba, lobis duplo latioribus quam longioribus emarginatis, lobulis orbicularibus antice rotundatis. Fructus $14 \mathrm{~mm}$ longi, $18 \mathrm{~mm}$ diametro. Semina $6 \mathrm{~mm}$ longa. - Arbor parva, fructibus viridibus (ex Broadw.).

Hab. in Trinidad: Bot. Gard. Herb. no 2462, ad Moruga road prope Eight Miles, m. Jun. fruct.: Broadway no. 2727. - Amer. austr.

Obs. $P$. grandifolius $L$, in speciminibus antillanis saltem, bene differt foliorum forma, pedicellis fl. fem. 5-15 mm longis, stigmatibus 3 basi connatis subquadratis v. ohtriangularibus, apice bifidis, lobulis triangularibus $v$. lanceolatis, fructibus $5-8 \mathrm{~mm}$ longis, $7-12 \mathrm{~mm}$ latis, seminibus $3,5-4,5 \mathrm{~mm}$ longis.

166. Phyllanthus monocladus Urb., spec. nov. (sect. Euphyllanthus).

Annuus. Caulis tenuis simplex $10-20 \mathrm{~cm}$ longus paullo compressus, linea $\theta$ foliis decurrente elevata notatus, glaber. Stipulae triangulares v. triangulari-lancolatae, non decurrentes, vix $0,4 \mathrm{~mm}$ longae. Folis distiche alterna, $0,5 \mathrm{~mm}$ longe petiolata, ovata $\mathrm{v}$, ovato-elliptica, basi 
rotundata, apice obtusa, 3-6 mm longa, 1,5-2,5 mm lata, penninervia. Flores masculi ad axillas $1-2$, vix $0,3 \mathrm{~mm}$ longe pedicellati. Sepala 5 libera, aequalia, obtriangularia, antice subtruncata, $0,6 \mathrm{~mm}$ longa. Filamenta basi vix connata; antherae 3 didymae horizontaliter rimosae. Flores feminei ad axillas solitarii, $0,3 \mathrm{~mm}$ longe pedicellati. Sepala 6 libera ovato-elliptica, apice obtusissima, $0,8 \mathrm{~mm}$ longa. Styli 3 . Ovarium glabrum. Capsula depressa, $2,7 \mathrm{~mm}$ diametro.

Radix brevis multiramulosa, Caules brunescentes, inferne nudi, superne foliosi, internodiis $2,5-5 \mathrm{~mm}$ longis. Foli a nervo medio prominulo, lateralibus paroissimis vix conspicuis. Flores masculi: Sepala quineuncialiter imbricata, membranacea, uninervia. Disci glandulae cum sepalis alternae obcordatae. Filamenta $0,1 \mathrm{~mm}$ longa. Flores feminei: Sepala sub fructu usque $1,3 \mathrm{~mm}$ longe accrescentia, ellipticooblonga, nervo medio crassiusculo obscure viridi, caeterum pallida. Disci glandulae optime evolutae, liberae, quadratae, apice truncatae. Styli $0,2 \mathrm{~mm}$ longi, basi connati, apice emarginato-bifldi.

Hab. in Trinidad in Piarco Savannah inter gramina, m. Dec. flor. et fruct.: Broadway no. 2130 .

Obs. Affinitatem inter Phylanthi species descriptas frustra quaesivi; ex antillanis $P$. heliotropus Wright et praesertin $P$. pruinosus Poepp. (e Cuba) maxime accedunt, e caeteris $P$. simplicicaulis Müll. Arg. (e Brasilia) et $P$. microphyllus H. B..K. (e Venezuela) multas notas similes pruebent.

167. Phyllanthus Elsiae Urb., spec. nov, (sect. Cicca).

Arbor sub anthesi aphylla, dioeca, glabra. Rami vetusti squamas tantum spiraliter dispositas triangulares gerentes, hornotini foliosi Stipulae triangulares acuminatae, non decurrentes, $1-1,5 \mathrm{~mm}$ longae. Folia distiche alterna, $1,5-2 \mathrm{~mm}$ tonge petiolata, breviter ovata usque orbicularia, basi subacutata v. rotundata, apice brevissime acuminata $v$. rotundata. $2-4,5 \mathrm{~cm}$ longa. $1,5-3,5 \mathrm{~cm}$ lata, penninervia, chartacea, integra. Inflorescentiae masculae in ramulis abbreviatis e ligno vetusto progredientibus plures, $1,5-2,5 \mathrm{~cm}$ longae, e glomerulis spicatim dispositis minutis compositae; bracteae triangulares pauciflorae; pedicelli filiformes 0,7-1,3 mm longi. Sepala 4 paullo imbricata cr. $1 \mathrm{~mm}$ longa, ambitu varia, bina late rotundato-rhomboidea, alterna anguste obovata, membranacea enervia. Discus non conspicuus. Stamina 4; flamenta basi ima cohaerentia $0,2 \mathrm{~mm}$ longa; antherae rotundatae paullo latiores quam longiores, intus supra basin affxae, loculis usque ad medium connective adnatic, superne liberis, lateraliter extrorsum et longitorsum dehiscentibus. Inflorescentiae fructiferae e ligno vetusto abeuntes $2-7 \mathrm{~cm}$ longae, caeterum ut in mare; pedicelli fruct. ex axilla bractearum $1-$ plures, $2-2,5 \mathrm{~mm}$ longi. Sepala 4 forma et magnitudine maris. Styli 3 basi in columnam perbrevem connati, fere ad basin bipartiti, ramis sublinearibus fere $2 \mathrm{~mm}$ longis iterum bifidis. Fructus globosi non sulcati, majores $6-7 \mathrm{~mm}$ diametro, 3-loculares, pericarpio $1,5 \mathrm{~mm}$ crasso crustaceo, non maturo, an serius capsulares? 
Arbor 6,6 m alta. Rami vetusti teretes, lenticellis crebris ovalibus $v$. orbicularibus et cicatricibus ramulorum delapsorum suborbicularibus obsiti, annotini in sicco angulati, capitulo ex hypsophyllis triangulariacuminatis conflato terminati. Rami hornotini euphylla gerentes usque $30 \mathrm{~cm}$ longi glaucescentes, internodiis $1-2 \mathrm{~cm}$ longis. Folia norvo medio supra subimpresso, lateralibus utroque latere 5-6 supra tenuiter, subtus paullo magis prominentibus et tenuiter reticulato-anastomosantibus, glaucescentia. Inflorescentiae non pedunculatae. Flores viriduli, odore dulci (ex coll.).

Hab. in Tobago prope Auchenskeoch beach, m. Mart. flor.: Broadway no. 4789 (typus), in Studley Park prope mare, $m$. Jul. fruct. immat praebens: Broadway no. 4557.

Obs. Species orn. Elsie Broadway, quae patrem in herbariis praeparandis adjuvit, dedicata, ex affinitate $P$. distichi (L.) Müll. Arg., qui prater alias notas floribus monoecis, disco evoluto, antheris rimas verticales offerentibus, fructu valde alieno 4-cocco longitrorsum profunde sulcato adhorret. Si subsectionem Ciccae novam propenere nolis, characteres subsectionis Euciccae emendandi sunt.

168. Croton microdon Urb., spec. nov.

Frutex dioecus. Rami hornotini lepidibus ferrugineo-brunescentibus circumcirca breviter multiradiatis adpressis induti. Stipulae minutao triangulari-lanceolatae cr. $0,7 \mathrm{~mm}$ longae, deciduae. Folia $3,5-0,5 \mathrm{~cm}$ longe petiolata, ovato-elliptica v. ovato-oblonga v, oblonga, basi rotundata v. subtruncata, $\theta$ parte inferiore sensim angustata, apice obtusiuscula, $5-9 \mathrm{~cm}$ longa, 1,5-4 cm lata, margine toto minute et subirregulariter dentata, nervis e basi sub-5, caeteris pinnatis, supra squamulis albidis, subtus squamulis crebrioribus brunescentibus multiradiatis adpressis vestite, sub basi ad petiolum glandulas binas breviter cylindraceas gerentia. Inflorescentiae (masculae tantum visae) terminales $5-10 \mathrm{~cm}$ longae, $1-1,5 \mathrm{~cm}$ longe pedunculatse; bracteae vix $1 \mathrm{~mm}$ longae valde deciduae. Flores e quaque bractea 2-plures; pedicelli $3-5 \mathrm{~mm}$ longi. Alabastra sphaeroidea $1,8 \mathrm{~mm}$ crassa lepidota. Sepala ovata. Petala oblonga villosula $2,5 \mathrm{~mm}$ longa. Stamina 14-15.

Rami vetustiores teretes, hornotini plus minus obtusanguli. Folia alterna, petiolis teretibus, nervo medio prominulo, lateralibus basalibus 2 interioribus in $1 / 2-1 / 2$ long. laminae ascendentibus, caeteris utroque latere 4-5. sub angulo cr. $50^{\circ}$ abeuntibus supra prominulis, subtus prominentibus et tenuiter conjunctis, denticulis apice incrassatis, chartacea, glandulis basalibus apice conaris. Inflorescentiae satis densiflorae, indumento ramorum. Flores musculi albi: Sepala 5 in aestivatione valvata, basi breviter connata. Petala in aestivatione parum imbricata, $0,5 \mathrm{~mm}$ lata, circumcirca villosa. Disci glandula $\theta$ subquadrata $\theta$, infern $\theta$ paullo latiores, apice truncato leviter emarginatao. Filamenta glabra; antherae juniores subquadratae, paullo latiores quam longiores.

Hab. in Sto. Domingo prov. Barahona prope Palo bonito $700 \mathrm{~m}$, m. Majo flor.: Fuertes no. 1582. 
Obs, Ex affinitate C. corylifolii Lam., qui colore indumenti, orenatura foliorum, monoecia et in forma typica forma foliorum discrepat.

169. Croton choristolopis Urb., spec. nov. - C. bixoides Müll. Arg. in DC. Prodr. XV. II (1866) p. 552 (quoad pl. Trinit, non Vahl).

Arborescens monoecus. Rami hornotini lepidibus sordide albidis circumcirca brevissime multiradiatis appressis dense induti. Stipula $\theta$ basi semicordata v. lanceolata subulato-acuminatae helicoidese v. foliorum summorum subulato-filiformes 5-7 mm longae. Folia 4-8 $\mathrm{mm}$ longe petiolata, ovata usque lanceolata, sed inferne semper latiora, basi breviter excisa, rotundata v. obtuss, apice plus minus longe acuminats, $\theta$ basi 5-nervia, utrinque argenteo-lepidota, lepidibus subtus numerosioribus, sed inter sese plane disjunctis, caeterum glabra, basi eglandulosa. Inflorescentiae in ramis et ramulis terminales. usque $10 \mathrm{~cm}$ longae, $0,5-1 \mathrm{~cm}$ longe pedunculatae, in $1 / \mathrm{t}-1 / \mathrm{s}$ inferiore feminese, caeterum masculae; bracteae lineares $1-2 \mathrm{~mm}$ longae deciduae 1-florae. Flores masculi 1,5-2 mm longe pedicellati. Alabastra obtuse 5-angula $2 \mathrm{~mm}$ crassa, circumcirca lepidota. Sepala triangulari-ovata. Petala latiusculo linearia, supra basin paullo angustiora ef barbata, caeterum breviter pilosa, 2,5 mm longa. Stamina 11-13. Flores feminei postremo $3-5 \mathrm{~mm}$ longe pedicellati, laxi. Sepala in $1 / 3$ long. inter sese connata, quoad libera late triangularia, sed lateribus et parte integra sub angulo sita supera solemniter reflexis. Petala nulla. Ovarium obtuse trigonum. Styll supra basin bis bifurcati, ramis omnibus ideo 12 .

Arbor parva, ligno desecto odorifero. Rami vetustiores teretes lenticellosi, hornotini angulati. Folia nervo medio vix impresso, basalibus 2 interioribus cr. usque ad medium productis, caeteris utrinque $3-4$ pinnatis grosse et tenuiter anastomosantibus, integra, supra viridia, subtus lepidibus argentea, membranacea v. chartacea. Flores masculi laxiusculi. Sepala 5 in aestivatione valvata, basi vix connata, $2,5 \mathrm{~mm}$ longa. Petala in aestivatione non imbricata, membranacea, $0,8 \mathrm{~mm}$ lata, 3-nervia. Disci glandulae basi contiguae, caeterum dissitae, rectangulares, apice truncatae, carnosulae. Filamenta usque $2,5 \mathrm{~mm}$ longe pubescentia; antherse rectangulari-ovales, longiores quam latiores. Flores feminei:' Disci glandulae confluentes. Styli $4 \mathrm{~mm}$ longi, usque supra medium pubescentes. Fructus juniores obtusissime trigoni, carpiḍiis supra stigmatum insertionem productis obtusissimis apice trifidi.

Hab. in Trinidad: Bot. Gard. Herb. no. 2440 (typus), Si ebor no. 269, prope Mayaro ad Radix Point, $m$. Jun. fruct. immat. ferens: Broadway no. 2642. - Margarita in El Valle, m. Jul. flor.: Miller ot Johnston no. 232.

Obs. I. Affines $C$ helicoideus Miill. Arg. et $C$. bixoides Vahl (ex insulis caribaeis, inter sese arcte propinqui nisi conspeciflci) foliis basi semper cordatis, facie infera lepidibus confluentibus tota argentea, pedicellis fructiferis $1-2 \mathrm{~mm}$ longis, sepalis flor. femin. fere ad basin liberis ovatis v. anguste ovatis, margine infero parum v. non recurvis, fructibus apice leviter emarginatis optime diversi sunt. 
Obs. Il. Sine ulla dubitatione specimina a ol. Mäller sub nomine C. bixoidis commemorata: Martinique ex herb. Pauché! et Trinidad leg. Sieber no. 269! speçies duas diversas praebent; descriptio ejus praesertim ad exemplar prius spectat.

Obs. III. Typus Vahlianus C. bixoidis, specimen ex herb. Surian no. 74 in herb. Jussieu no. 16334 (verisimiliter $\theta$ Martinique, qua in insula praeter in Haiti Surian cum ill. Plumier collectiones botanicas fecit), ut cl. Lecomte communicavit, satis cum exemplaribus martinicensibus Dussianis convenit, sed non cum Sieberiano no. 269 (e Trinidad).

170. Croton claraēnsis Urb., spec. nov.

Frutex monoecus. Rami hornotini squamulis flavidis v. sordide flavidis v. cinerascentibus circumcirca multiradiatis flocculosis dense induti. Stipulae nullae. Folia cr. $1 \mathrm{~mm}$ longe petiolata, anguste linearia aequilata, basi rotundata, apice obtusa $v$. rotundata, $2-5 \mathrm{~cm}$ longa, 1,8-3 mm lata, supra nitida, subtus obscura, nervo medio supra profunde sulcato-impresso, subtus crasse prominente, lateralibus supra numerosis subhorizontalibus impressis, subtus nullis, supra glabra laevia, subtus squamulis multiradiatis dense tomentosula, basi non glandulifera. Inflorescentiae terminales $1-2 \mathrm{~cm}$ longae, non $v$. usque $5 \mathrm{~mm}$ longe pedunculatae, ad basin femineae, caeterum masculae; bracteae lineares cr. $1 \mathrm{~mm}$ longae, apice spicae alabastra paullo superantes. Flores e quaque bractea solitarii sessiles. Flores masculi: Alabastra globulosa, $1,8 \mathrm{~mm}$ crassa, tomentosula. Sepala anguste ovata. Petala obovatospathulata, inferne sensim angustata, supra basin longiuscule barbata, caeterum glabra, $2 \mathrm{~mm}$ longa. Stamina cr. 14-16. Flores feminei: Sepala ovato-lanceolata. Petala nulla. Ovarium sphaeroideum. Styli supra basin ipsam bifidi, ramis integris.

Rami vetustiores teretes glabrati, punctulis minutis obsessi, hornotini teretiuseuli. Folia lateribus limbi convexis, integra, supra nitida in sicco glaucescentia, subtus obscure flavida, coriacea, fragilia. Flores masculi dense aggregati. Sepala 5 in aestivatione valvata, basi breviter connata, $2 \mathrm{~mm}$ longa. Petala in aestivatione non imbricata, membranacea, superne $\mathrm{cr}$. $0,6 \mathrm{~mm}$ lata, 3-nervia. Disci glandulae 5 contiguae breviter obcordatae carnosae. Filamenta $1,7 \mathrm{~mm}$ longa, glabra; antherae ovales, longiores quam latiores. Flores fominei ad basin rhacheos perpauci. Disci glandulae valde abbreviatae in annulum connatae. Styli $2 \mathrm{~mm}$ longi pilis stellaribus villosuli.

Hab. in Cuba prope erbem Santa Clara in palmeto, m. Mart. flor.: N. L. Britton, E. G. Britton, P. Wilson no. 6047.

Obs. Afflnis C. Sagraearo Müll. Arg., qui foliis superne sensim angustatis, basi biglandulosis, nervis lateralibus sub angulo 50-60 abeuntibus, inflorescentiis elongatis, floribus femineis numerosis, bracteis perparvis inter alabastra mascula non prominentibus, floribus masculis bene pedicellatis recedit. Paullo longius distat $C$. linearis Jacq. habitu similis. 
171. Adelia macrophylla Urb., spec. nov. - ? Ricinella pedunculosa (A. Rich.) Müll. Arg. lusus grandifoliws Müll. Arg. in DC. Prodr. XV. II (1866) p. 730. - Adelia ricinella Pax! in Pflanzenr. 63. Heft (IV. 147. VII a. 1914) p. 66 (p.'p., non L.).

Arbor parva. Rami non spinescentes glabri. Folia 2-6 mm longe petiolata. rhombea, obovata usque oblonga, semper superne latissima, inferne sensim et satis longe cuneatim angustata, basi ipsa obtusa et obsolete emarginata, apice obtuse acuminata, $7-11 \mathrm{~cm}$ longa, $2,5-5,5 \mathrm{~cm}$ lata, nervo medio supra solemniter, lateralibus tenuiter prominentibus, non pellucido-punctata, domatiis exceptis glabra. Flores masculi ad axillas pauci glomerati; pedicelli $2-3 \mathrm{~mm}$ longi, breviter pilosuli. Alabastra globulosa tomentosa. Sepala 5, rarius 4, ovala v. ovato-lanceolata $2,5 \mathrm{~mm}$ longa. Discus annularis brevissime pilosus. Stamina 10-13; flamenta supra basin inter se connata, usque $2,5 \mathrm{~mm}$ longa; antherae quadratae v. quadrato-crbiculares; staminodia intus adjecta $4-5$ filiformia glabra flamentis duplo breviora. Ovarii rudimentum nullum. Flores feminei mihi non obvii. Pedicelli fructiferi $2,5-3,5 \mathrm{~cm}$ longi. Sepala 6 , linearia v. lanceolatu-linearia $2,5 \mathrm{~mm}$ longa. Capsula $12 \mathrm{~mm}$ diametro, $8 \mathrm{~mm}$ longa, brevissime pilosa. Semina globosa grisea $4 \mathrm{~mm}$ diametro.

Hab. in Tobago in Spring Garden, m. Fobr. flor., m. April. fruct.. 13 roadway no. $3491,3573$.

Obs. Ab $A$. ricinella $\mathrm{L}$. valde affini et per Antillas a Cuba usque ad Curaçao divulgata ramis inermibus, foliis multo amplioribus, capsulis seminibusque majoribus facile distinguenda nec formis transitoriis cum illa conjuncta.

Anm. Ich fand hier innerhalb der Staubblätter 4-5 fädliche kahle Organe, die unmöglich als 0varium rudimentarium angesprochen werden können, sondern nach Ausbildung und Zahl wohl sicher Staminodien sind. Ähnliche Gebilde, auch 4-5 an Zahl, zeigte $A$. ricinella L.; hier sind sie aber viel kürzer, dreieckig bis lanzettlich, selten eins oder das andere fädlich; sio gehen deutlich aus dem obersten, Rande der Staubblattsäule $a b$, bilden hier einen Wirtel, und führen zwischen sich keine Spur eines Ovarrudimentes. Danach ist die Angabe von Pax I. c. p. 65: Ovarii rudimentum 3-partitum zu berichtigen.

172. Acalypha angustifolia Sw.! Prodr. (1788) p. 99 et Flor. II p. 1174; Spreng. Syst. III p. 882; Müll. Arg.' in Linnaea XXXIV p. 22 et in DC. Prodr. XV. II p. $387^{-}$(cum var. genuina ot glabrata Müll. Arg.). - $\dot{A}$. domirgensis Śpreng.! Syst. III (1826) p. 880. - A. carpinifolia Müll. Arg.! in Linnaea XXXIV (1865) p. 22 ot in DC. Prodr. XV. II p. 836 (cum var. genuina et domingensis Müll. Arg., an Poir.?). - Plum. ed. Burm. p. 165 tab. 172 fig. ad sinistram super.

Suffrutex v. frutex $0,7-2 \mathrm{~m}$ altus, valde variabilis, nunc (in specim. Swartz.) pubescens, nunc (in specim. caeteris) subglaber, follis linearibus v. lanceolatis v. ovato-lanceolatis, sed semper supra basin $v$. inferne latissimis, basi ipsa emarginatis v. obtusis, nervis lateralibus nunc parcioribus et arduis (in specim. Swartz. et Buch.), nunc crebrioribus et minus 
arduis (in ic. Plum. et exempl. caeteris), bracteis femineis plus minus evolutis et plus minus profunde 5-9-fidis. Flor. Jan., Apr., Jul., Aug.

Hab. in Haiti: C. Ehrenberg, Picarda no. 49, 362, 444, Poiteau (ex Müll. Arg.), locis saxosis ad rivulos: Swartz (mus. Holm.), prope Marmelade: Nash et Taylor no. 1286, prope Plaisance in faucibus umbrosis $400 \mathrm{~m}$ : Buch no. 1064, prope Port-au-Prince in sylvis montanis: Bertero no. 1045, Jacquemont, in Fond Parisien: Picarda no. 305, in Morne Faure latere septentr. in faucibus humidis: Christ no. 1836. Sto. Domingo: Bertero, in prov. Barahona prope Maniel et Paradis ad rivulos, vias et in sylvis $100-200 \mathrm{~m}$ : Fuertes no. 452, 1012, $1012 \mathrm{~b}$, v. Türckheim no. 2756 .

Obs. An A. carpinifolia Poir. in Lam. Enc. VI (1804) p. 203 (cond. in Plum. ed. Burm. tab. 172 f. 1) ad formas A. angustifaliae Sw, pertineat, anne speciem propriam praebeat, specimine congruente nune mihi non obvio dicere nequeo. Ill. Plumier ipse in Cat. Plant. amer. (1703) p. 20 ambas species sub nominibus Masihot ulmi folio ampliore ot Manihot ulmi folio angustiore soparavit.

173. Acalypha hispaniolac Urb., spec. nov.

Monoeca. annua v. fruticulosa, $15-25 \mathrm{~cm}$ alta, Radix parum v. non incrassata. Caules supra basin radicantes, arcuato-erecti; rami hornotini pilis brevibus patentibus v. curvulis satis dense vestiti. Stipulao fliformes v. subulato-fliformes, 3-5 mm longae. Folia 20-2 mm longe petiolata, ovata v. breviter ovata, basi truncata v. leviter cordata, apice obtusiuscula v, obtusa, $2-4 \mathrm{~cm}$ longa, $1.5-3 \mathrm{~cm}$ lata v. suprema minora, margine toto crenata v. serrata, $\theta$ basi 5-nervia, nervis supra tenuiter $v$. vix, subtus magis prominentibus et reticulato-anastomosantibus, supra glabra v. parce pilosa, subtus ad nervos tantum v. etian in facio pubescentia, chartacea, plerumque subtus purpurascentia. Inflorescentiae terminales plerumque bisexuales, ad basin $v$. inferne femineae, caeterum masculae, raro unisexuales, nunc masculae nunc femineae, floribus singulis femineis in axillis foliorum superiorum fere semper additis; pars mascula usque $5 \mathrm{~cm}$ longa, 2-2,5 mm crassa, glomerulis contiguis $v$. infimis inter sese disjunctis, bracteis filiformibus $1-1,5 \mathrm{~mm}$ longis suffultis; feminea nunc 1-, nunc pauci-, nunc pluriflora, postremo usque $7 \mathrm{~mm}$ crassa. Alabastra mascula $0,3-0,4 \mathrm{~mm}$ crassa, obtuse 4-gona. Bracteae fl. fem. explanatae suborbiculares, fere usque ad medium pinnatifidae, lobis 9-13 triangulari-lanceolatis acutis. Styli usque $7 \mathrm{~mm}$ longi. multipartiti purpurej. Capsulae sphaeroideae, superne hirsutae. Semina ovata, 1,2 $\mathrm{mm}$ longa, 0,9 $\mathrm{mm}$ crassa, pallide brunescentia.

Hab. in Haiti prope Furcy: Picarda no. 1026, prope La Chapelle du Morne Faure $1270 \mathrm{~m}$ alt. locis humidis: Christ no. 1833. - Sto. Domingo prope Constanza in pinetis $1190 \mathrm{~m}$ alt. et prope Maniel de 0 cos in fruticetis $300 \mathrm{~m}$ alt.: v. Türckheim no. 3145 (typus), 3689 , in prov. Barahona in montibus et ad rivulos 1000-1300 m alt., inter Las Salinas et Arroyo Montaña in sylvis $500 \mathrm{~m}$ alt., prope Bahoruco in humidis $75 \mathrm{~m}$ alt. Fuertes no. 538, 607, 1008, 1441, 1442. 
Obs. Aftinis A. chamaedryfolia (Lam.) Müll. Arg. (Plum. od. Burm. tab. 172 f. 2) foliis praesertim superioribus plerumque angustioribus oblongis, sed supra basin latissimis nunquam purpurascentibus et imprimis bracteis dentatis, dentibus limbo integro duplo et ultra brevioribus facile distinguenda.

174. Euphorbia parolitora Urb., spec. nov.

Radix crassa lignosa perennis, sub collo usque $4 \mathrm{~mm}$ crassa. Caules - caudice plures usque $26 \mathrm{~cm}$ longi glaberrimi obscure purpurascentes, supra basin $0,8-1,5 \mathrm{~mm}$ crassi, ramosi, arcuato-erecti internodiis $2-4 \mathrm{~cm}$ longis. Stipulas antice in lacinias setaceas dissectao v. lanceolatao et incisae. Folia opposita, $1-1,5 \mathrm{~mm}$ longe petiolata, infima oblique subrotunda, catera rhomboidea v. oblique ovats, basi obliqua subtruncata v. obsolete cordata, apice obtusa v. plerumque acuta, $\mathbf{5}-\mathbf{9} \mathrm{mm}$ longa, 1-7 mm lata, ineequilatera, nervo medio supra subimpresso, lateralibus inferne parum conspicuis, margine angustissime recurvo ad latus longius fere usque ad baskin, ad latus brevius usque supra medium argute serrata, pergamacea. Involucra in apice ramorum et ramulorum solitaria parca, 1-2,5 mm longe pedicellata, semiovalia, 1,3 $\mathrm{mm}$ longa; lobi primarii triangulares pilosuli; glandulae 4 transversim ellipticas; appendices in eodem flore inaequimagnae semiorbiculares usque semilunares glandulis aequilatae $v$. paullo latiores et longiores, in nonnullis receptac. valde reductae. Styli $0,8 \mathrm{~mm}$ longi, tore usque ad basin bipartiti. Capsulae integrae non visae; valvae $1,9 \mathrm{~mm}$ longae, dorso obtuse subcarinatae. Semina ignota.

Hab. in Haiti prope Marmelade, m. Aug. flor.: Nash ot Taylor no. 1269 .

Obs. Ex affinitate $E$. Preslii (auss. et $E$. hyssopifoliae L., quae foliis et inflorescentiis discrepant.

\section{Prlamosia Urb.}

\section{(genus novum Flacourtiacearum).}

Flores dioeci, masculi tantum visi. Sepala 4 libera squamiformia leviter imbricata membranacea. Petala nulla. Discus annularis. Stamina 4 intra discum inserta, cum sepalis alterna, inter sese libera; flaments brevia; antherae intus sub medio affxae, orbiculari-globulosae, biloculares birimosae, loculis extrorsum longitudinaliter dehiscentibus; pollinis granula tririmosa laevia. Ovarii rudimentam bene evolutum superum liberum conico-lineare, apice integrum. - Frutex domingensis, spinis axillaribus simplicibus, ligno non amaro; rami hornotini pulverulento-pilosi. Stipulae minutissimae inter sese aequales. Foli s spiraliter disposita, breviter petiolata, pennatinervia, superne crenata, coriacea. Flores e gemmis squamosis solitarii, minuti, brevissime pedicellati.

Obs, Ex affinitate Xylosmae, quae floribus masculis polyandris ovario rudimentario carentibus (et aliis notis?) diversa est, ot Azarae (e Chile et Argentina), praesertim A. microphyllac Hook. f., quae ramis inermibus, ligno amaro, stipularum phyllo uno v. utroque euphylloideo-ampliato, 
inflorescentiis racemosis v. corymbosis (an etiam floribus femineis fructibusque?) recedit. - Priamos, rex Trojanorum, nomen generis mihi exhibuit.

175. Priamosia domingensis Crb., spec. nov.

Frutex $4 \mathrm{~m}$ altus. Rami novelli parce pulverulento-pilosi, vetustiores lenticellis parvis rotundis obtecti; spinae $3-8 \mathrm{~mm}$ longae, in axillis gemmam e squamis lanceolatis compositam v. rosulam foliorum gerentes. Stipulae triangulares cr. $0,2 \mathrm{~mm}$ longae. Folia $1-1, \tilde{\mathrm{j}} \mathrm{mm}$ longe peticlata, ovata v. obovata, basi acutata v. cuneata, antice obtusissima v. rotundata, $1-2 \mathrm{~cm}$ longa, $0,6-1,3 \mathrm{~cm}$ lata, nervo medio supra subimpresso, lateralibus praesertim subtus conspicuis crebris, hornotina tenuia in sicco nigricantia vetustiora coriacea supra glauca. Flores masculi e coma squamarum parum exserti, pedicello $1,3 \mathrm{~mm}$ longo insidentes. Sepala ovato-triangularia, antice acutata, margine supero flmbriato-cilista, supra basin utrinque dentem solitarium gerentia, pallida, ad apicem bruneo-colorata et paullo incrassata, $1 \mathrm{~mm}$ longa. Filamenta ci. $1.5 \mathrm{~mm}$ longa; antherae $0,6 \mathrm{~mm}$ diametro.

Hab. in Sto. Domingo in Valle de Constanza $1170 \mathrm{~m}$ alt., m. Majo flor.: Eggers no. 2285.

176. Plinia L. Syst. I ed. (1735) et Gen. I ed. (1737) p. 155 no. 438 $=$ Plinia Plum. Nov. Plant. Gen. (1703) p. 9 tab. 11 fig. super. - Marlierea Camb. in A. Saint-Fil. Fl. Bras. mer. II (1829) p. 373.

Diese von Plumier aufgestellte, von Linné übernommene und anerkannte Myrtaceen-Gattung ist von allen Autoren entweder übersehen oder falsch gedeutet worden; auch mir ist die Plumiersche Abbildung der Blüten und Früchte, zu der die Editio Burman 1759 auch das Habitusbild brachte, bei der Bearbeitung der westindischen Myrtaceen 1895 entgangen. Es kann gar keinem Zweifel unterliegen, dass die Plumiersche Art, die wohl sicher von Martinique stammt, mit Marlierea glomerata Berg identisch ist. Blätter (naürlich nicht gefiedert, sondern gegenständig), Blüten und die eigenartigen Früchte stimmen vollständig überein. In der Zeichnung bei Burman gehen freilich die Blüten einzeln aus dem älteren Holze hervor, während sie in Wirklichkeit zu mehreren einen kleinen Knäuel bilden. Ähnliche Freiheiten hat sich Plumier wiederholt gestattet; in diesem Falle würde die Abbildung bei einer genauen Darstellung wohl an Leutlichkeit verloren haben.

Es gewährt mir eine ganz besondere Befriedigung, dieser dem römischen Naturforscher Plinius secundus zu Ehren benannte Gattung wieder zu ihrem Rechte verholfen zu haben; sie wird vor anderen die Priorität behalten und bestehen bleiben, wie man auch über die sehr schwierige Abgrenzung der Myrtaceen-Gattungen in Zukunft urteilen mag.

Die von dem Typus der Gattung hergenommenen wesenllichen Charaktere, welche nach dem monographischen Studium anderer hierher gehöriger Arten zu einer zutreffenden Genus-Diagnose erweitert werden mögen, sind die folgenden: 
Flores ad nodos ligni vetustioris in glomerulum pluriflorum collecti arcte sessiles, bracteis rotundatis circumdati, 4-, raro 5-meri. Calycis tubus breviter turbinatus, supra ovarium ratione satis longe productus, in alabastro paene clausus, apicibus sepalorum tantum liberis valvatis, sub anthesi in lobos inaequales profundius disruptus. Petala parva. Stamin a numerosa pluriseriata, libera; flamenta in aestivatione inflexa, filiformia; antherae versatiles, loculis parallelis /ongitudinaliter dehiscentibus. Ovarium 2-loculare; stylus fliformis, stigmate parvo; ovula in loculis 2. Bacca sphaeroidea, apice cupula calycis disciformi obsessa (15-18 mm longa, $20-25 \mathrm{~mm}$ diametro), plus minus manifeste 12-costata, costis nunc sub epicarpio plicato absconditis. Semen solitarium subcompressum, testa endocarpio adhaerente. Cotyledones liberae, intus planae, dorso convexae, ita dispositae, ut altera ad baccae apicem, altera ad ejus basin versus sita sit, aequales v. subinaequales; radicula brevissima, sed manifesta mammiformis. - Arbor parva erecta gracilis.

Von westindisehen Arten gehören wohl mit Sicherheit zu dieser Gattung:

Plinia pinnata L. Spec. I ed. I (1753) p. 516 et II ed. I p. 735. $P$. crocea L. Mant. II (1771) p. 244 in obs. - P. pentapetala L. L. c. p. 402. - Marlierea glomerata Berg! in Linnaea XXVII (1855) p. 14; Urb. in Engl, Bot. Jahrb. XIX p. 589; Duss Flor. Ant. franç. p. 267. Myrciaria Trinitatis Berg in Linnaea XXVII (1856) p. 336 (ex descr. et numero Sieber.). - Stenocalyx Plumieri Berg! in Linnaea XXX (1861) p. 698. - Eugenia Plumieri Niedenzu! in Engl.-Prantl Nat. Pflanzenfam. IIl. 7 (1893) p. 82. - Plinia fructu croceo odorato Plum. Gen. (1703) p. 9 tab. 11 et ed. Burm. p. 219 tab. 225.

Hab. in Martinique: Duss no. 659, Hahn no. 1087 (hb. Kew.), lsert (mus. Haun.), Plée no. 727, L. C. Richard. - St. Lucia: Anderson (hb. Kew.). - Trinidad: Sieber no. 151. - Guiana anglica: Rich. Schomburgk.

Plinia cubensis (Griseb.) Urb., comb. nov. - Calycorectes cubensis Giriseb. Cat. cub. (1866) p. 90. - Marlierea cubensis Krug et Urb. in Engl. Bot. Jahrb. XIX (1895) p. 589.

Hab. in Cuba: Wright no, 2435.

Plinia Dussil (Krug et Urb.) Urb., comb. nov. - Marlierea Dussii Krug et Urb. in Engl. Bot. Jahrb. XIX (1895) p. 590; Duss Flor. Ant. franç. p. 267.

Hab. in Portorico: Sintenis no. 4315. - Guadeloupe: Duss no. $-2750,3808,3810,4000,4010$. - Martinique: Duss no. $4000 \mathrm{~b}$, 4032.

Obs. Marlierea Sintenisii Kiaersk. (e Portorico: Sintenis uo. 1476) et M. Guildingiana (Griseb.) Krug et Urb. (e St. Vincent: Guilding H. H. et G. W. Smith no. 1735,1738 et Grenada: Broadway no. 1488 , 1901) ob inflorescentias abhorrentes in statu fructifero quoad characteres embryonis iterum examinandae et cum descriptione mea typi generis comparandae sunt. 
177. Chrysophyllum Immoreum Urb., spec. nov.

Rami juniores pilis adpressis pallide ferrugineis induti. Folia 7-10 mm longe petiolata, ovalia, elliptica usque elliptico-oblonga, basi scuta v. rarius obtusiuscula, antice acuminata, apice ipso obtuso, $5-11 \mathrm{~cm}$ longa, 2,5-5 cm lata, nervis lateralibus supra tenuiter v. vix prominulis, subtus prominentibus, supra nitida in sicco olivacea, subtus pube breyi initio pallide ferruginea, mox albescente, demum decidua obsessa. Pedicelli fructiferi solitarii, 5-6 mm longi. Stigmata 6-7. Fructus sphaeroidei, multo crassiores quam longiores, apice solemniter immerso quasi excavati, majores (immaturi), qui adsunt, $10 \mathrm{~mm}$ longi, $13 \mathrm{~mm}$ crassi.

Arbor. Rami vetustiores lenticellis crebris parvis subrotundis obsessi teretes, hornotini subangulati, internodiis $1,5-2 \mathrm{~cm}$ longis. Folia petiolis $1-1,5 \mathrm{~mm}$ crassis supra profunde sulcatis, nervo medio supra solemniter impresso, lateralibus utroque latere $8-10$ sub angulo $50-60^{\circ}$ abeuntibus, supra non, subtus obsolete anastomosantibus, margine plana, coriacea. Sepala 5 suborbicularia dorso breviter ferrugineo-pilosa. Fructus inferne brevissime adpresseque pilosi.

Hab. in Tobago prope Easterfield, m. Jan. fructus immat. praebens: Broadway no. 4411.

Obs. Inter species affines (e. gr. Ch. oliviforme L.) forma fructuum statim recognoscendum.

178. Marsdenia maculata Hook. in Bot. Mag. LXXIII (1847) tab. 4299; Griseb. Flor, p. 422: Schlechter in Urb. Symb. I p. 274. - M. elliptica Griseb.! Kar. in Abh. Ges. Wiss. Götting. VII (1857) p. 241 (93) (non Dcne.); Duss Flor. Ant. franç. p. 400. - M. fusca Schlechter 1. c. (1899) p. 274 (quoad plant. Guad. et Mart., nec Wright). - Apocynum majus scandens, siliqua oblonga, tumida et glabra Plum. Descr. Plant. Amér. (1693) p. 81 tab. 95. - Liane laicteuse Ant. gall. (ex Plum.), Liane à vacho Mart. (ex Duss).

Folia basi obtusissima, rotundata v. subtruncata, anguste excisa. Sepála rotundata $\mathrm{v}$. breviter ovata, $4 \mathrm{~mm}$ longa. Corolla $7 \mathrm{~mm}$ longa; lobi tubo duplo et ultra longiores.

Hab. in Saba?: Suringar (sterilis). - Guadeloupe: Bertere no. 391, Duchassaing. - Martinique ad Case-Pilote in montibus siccis in fruticetis püssim: Duss no. 1857 (sed vix floribus albis gaudens). - Insulae gallica e, m. Jun., Jul. flor., m. Mart., April. fruct.: ex P lu mier (Alores rubro-brunei). - Tobago in Lot 42, m. April. flor.: Broadway no. 4468. - Trinidad: Lockhart (ex Hook.), Purdie (ex Griseb.), prope Blanchisseuse, $m$. Majo flor. et fruct.: Broadway no, 2253 (flores sanguinei odori; fructus viridis; succus lacteus copiosus). - Margarita in El Valle, m. Jul., Aug. flor.: Miller et Johnston no. 17, Johnston no. 67. - Panama, Columbia, Venezuela (ex Griseb.).

Obs. M. fusca Wright (e Cuba) foliis basi obtusis v. rotundatis, non v. levissime et late subcordatis, sepalis ovatis v. anguste ovatis $5-6 \mathrm{~mm}$ longis, corolla $8 \mathrm{~mm}$ longa, lobis tubo aequilongis diversa est. 
Anm. Die zitierte Plumiersche Tafel war bisher von niemanden zu deuten versucht worden.

179. Wigandia urens (L.) Urb., comb, nov. (non H. B. K. 1818, nec Cham. et Schlecht. 1831, nec Choisy 1833, nec Benn. 1871). - Nicotiana urens L. Syst. X ed. II (1759) p. 932 ot Spec. II ed. I p. 259; Poir. in Lam. Enc. IV p. 480. - Wigandia pruritiva Spreng. Syst. I (1825) p. 865. - W. crispa Choisy in DC. Prodr. X (1846) p. 184 (quoad pl. Doming.); Brand in Pflanzenr. 59. Heft (IV p. 251) (1913) p. 135 (quoad syn. Plum., non H. B. K.). - W. reflexa Brand 1. c. - Nicotiana arborescens, spinosissima, flore excalbido Plum. Cat. pl. amer. (1703) p. 3 et ed. Burm. p. 204 tab. 211. - Pringa moza Doming.

Flores albi, m. Mart., Aug., Dec.

Hab. in Cuba prope Santiago in Pinal de Nimanima: Linden no. 2107. - Haiti: Picarda no. 113. - Sto. Domingo in prov. Barahona prope Barahona ad rivulos: Fuertes no. 9, prope Paradis ad Rio Nizaito inter fluvii lapides: v. Türckheim no. 2778, propo Alpargatal in litore Rio Yaque: Fuertes no. 885.

Anm. Brand übersah in seiner monographischen Bearbeitung der Hydrophyllaceen, dass die Plumiersche Art bereits von Linné einen Namen erhalten hatte, und reihte sio irrtumlicherweise unter $W$. crispa (R. et P.) H. B. K, ein, eine Art, die nur aus Peru und Ecuador békannt ist und sich von der unsrigen auf den ersten Blick schon durch die doppelt grösseren Kelche unterscheidet. Vielleicht liess er sich bei dieser Identifizierung dadurch täuschen, dass auch die Abbildung Plumiers recht grosse Kelche aufwejst. Wer aber die Plumierschen Zeichnungen genauer kennt, weiss, dass dieser sonst so sorgfältige Beobachter mit Vorliebe die Bläten oder Blütenteile grösser darstellte, als sie in Wirklichkeit waren; das zeigen auch die Exemplare von Hispaniola, wo Plumier, der nur auP den Antillen reiste, unzweifelhaft seine art gezeichnet und studiert hat, Infolgedessen beschrieb Brand die Cubaund Hispaniola-Pflanzen als neue Art: $W$. reflexa. Es ist nun zu bodauern, dass bereits eine andere Art dieser Gattung (mit 3-4 mal kleinerer Blumenkrone), die ursprünglich (1802) als Hydralea urens von Ruiz und Pavon aufgestellt war, durch Überführung au Wigandia den Namen $W$. urens Choisy (1833) erhalten hat; ausserdem existieren auch noch 3 andere Arten, die von den Autoren als $W$. urens aufgeführt worden sind. Die Hydralea urens Ruiz ot Pav. muss demnach einen anderen Namen: $W$. hispida (Pav.) Urb. erhalten. Wor aber die alte Nomenclatur beibehalten will, hat für $W$. reflexa Brand den Namen $W$. pruritiva Spreng. anzunehmen, weil dieser sich auf die Linnésche Spezies gründet. 